\title{
Référents, Sens Et Effets De Sens De L'énoncé «Vieux Père, Voilà Ton Petit / Tes Petits » Dans Le Français De Côte d'Ivoire
}

\author{
Gnato Sia Modeste \\ Université Félix Houphouët-Boigny, Abidjan-Cocody, Côte d'Ivoire
}

Doi:10.19044/esj.2018.v14n20p202 URL:http://dx.doi.org/10.19044/esj.2018.v14n20p202

\begin{abstract}
In Côte d'Ivoire, as elsewhere in the French-speaking world, the French language is characterized by a constant movement on the lexical and semantic level. This situation, which testifies to the dynamism of French in a foreign context, is a constant source of thought for linguists. The present study focuses on the statement Old Father, this is your little one, a recurring expression in the Ivorian conversational landscape. Using the tools and methods of structural grammar and grammar of meaning, the analysis brings out the referential dimension, the meaning and the sense effects subsequent to the use of this utterance.The study shows that, contrary to the standard meaning which indicates a simple presentation of individuals in the same discursive environment, in the Ivorian context, the statement evokes two opposite social classes: on the one hand, the category of old fathers and the other that of the little ones. In addition, the statement covers several values: the comparison of two social classes involved, the evocation of a fake filiality, the axiological values of self-pity and of request.
\end{abstract}

Keywords: Ivorian context, language practice, referents, meaning, effects of meaning

\section{Résumé}

En Côte d'ivoire, comme ailleurs dans la francophonie, la langue française se caractérise par une mouvance constante sur le plan lexical et sémantique. Cette situation, qui témoigne du dynamisme du français dans un contexte étranger, donne en permanence matière à réflexion aux linguistes. La présente étude se focalise sur l'énoncé Vieux père, voilà ton petit/tes petits, une expression récurrente dans le paysage conversationnel ivoirien. Recourant aux outils et méthodes de la grammaire structurale et de la grammaire du sens, l'analyse fait ressortir la dimension référentielle, le sens et les effets de sens subséquents à l'usage dudit énoncé. L'étude montre que, contrairement au 
sens standard qui indique une simple présentation d'individus dans le même environnement discursif, dans le contexte ivoirien, l'énoncé évoque deux classes sociales opposées : d'un côté, la catégorie des vieux pères et de l'autre celle des petits. De plus, l'énoncé recouvre plusieurs valeurs : la comparaison de deux classes sociales impliquées, l'évocation d'une filialité factice, les valeurs axiologiques de l'auto-apitoiement et de requête.

Mots clés: Contexte ivoirien, pratique langagière, référents, sens, effets de sens

\section{Introduction}

Ancienne colonie française, la Côte d'Ivoire a hérité de la langue française depuis l'époque de la colonisation comme tous les pays francophones de l'Afrique de l'ouest. A cette période, la langue française était, entre autre, utilisée pour les besoins administratifs du moment. Ce médium servait de rapprochement entre la population indigène et les colons, étant donné la pluralité linguistique des peuples africains. Adoptée comme langue officielle au lendemain des Indépendances en 1960, le français devient le principal moyen de communication en Côte d'ivoire étant donné que «les langues locales n'ont pas été développées pour assumer le rôle de langue nationale » (H. Lagerqvist, 2001 :119). Ainsi, « même les ouvriers sur les chantiers, les petits employés /.../ sont obligés de communiquer entre eux ou avec leurs patrons dans une langue qu'ils ne maîtrisent qu'imparfaitement »(J. N. Kouadio, 2008 :184). Cette situation linguistique va générer une disparité au niveau des utilisateurs de ce véhiculaire, tous les usagers n'ayant pas eu la même durée et la même qualité de l'enseignement du français. Le résultat des études menées, à ce sujet, par Hans Lagerqvist (2001 :19), fait état de l'existence de trois catégories de locuteurs. La première regroupe ceux qui utilisent le français de type basilectal ; la seconde concerne ceux du type mésolectal et la dernière est du type acrolectal ${ }^{1}$. Les locuteurs des groupes basilectal et acrolectal n'ayant pas une bonne maitrise du français, leurs productions langagières se caractérisent par une mouvance lexicale et sémantique. «Certains mots, locutions et tournures sont si expressifs qu'ils finissent par paraître emblématiques de cette façon toute ivoirienne de jouer avec la langue française » écrit à ce propos H. D. Bohui, (2015 : 8). Cet

\footnotetext{
${ }^{1}$ - Le français basilectal est celui des locuteurs non scolarisés ou ceux qui ont arrêté les études au cycle primaire pour diverses raisons.

- Le francais acrolectal désigne le parler des intellectuels ivoiriens. C'est ceux qui ont fait des études universitaires et qui ont donc une parfaite maîtrise de la langue française.

- Le français mésolectal fait allusion au parler des ivoiriens qui ont arrêté leurs études dans l'enseignement secondaire à telle enseigne qu'ils n'ont pas une connaissance parfaite de la pratique des règles syntaxiques de la langue française.
} 
univers donne constamment matière à réflexion aux grammairiens-linguistes pour analyser les phénomènes de décatégorisation-recatégorisation et de conversions sémantiques des syntagmes usités. L'étude de l'énoncé vieux père, voilà ton petit / tes petits s'inscrit dans cette optique. Il s'agit d'une expression récursive dans les actes locutoires des sujets ivoiriens catégorisés comme usagers du français basilectal et, pour une partie, mésolectal. Progressivement, l'énoncé va faire son bonhomme de chemin au point de se nationaliser et même de s'internationaliser. En faisant de cette suite le point focal de la présente étude, notre objectif consiste à mener une analyse descriptive et interprétative dudit énoncé afin de faire ressortir ses "'ramifications" sémantiques. En termes plus explicites, à quoi renvoient les groupes syntagmatiques vieux père et petits dans le contexte ivoirien ? De plus, quelles sont les intentions des locuteurs impliqués, le sens et les effets de sens subséquents à l'usage de l'expression ? Telles sont les interrogations principales autour desquelles s'articule la présente étude. Répondre à ces différentes préoccupations nécessite que l'on établisse un cadre théorique et méthodologique adéquat pour mener une étude descriptive et interprétative permettant de circonscrire tous les faisceaux sémantiques de l'énoncé.

\section{Cadre théorique et méthodologique}

\subsection{Section théorique}

En évoquant la notion de cadre théorique à cette étape du travail, nous entendons nous inscrire dans la perceptive conceptuelle de C. Gohier (2000). Pour cet auteur, "par cadre théorique, on entend la " matrice théorique " qui donne des assises à la recherche. Il s'agit de balises théoriques issues de l'examen des théories /.../ qui cernent l'objet de l'étude » (C. Gohier, 2000 : 102). Du fait de l'importance de cette étape dans tout travail de recherche scientifique, elle est comparée au choix de «matériaux de construction /.../ qui déterminent comment les parties de la maison seront assemblées » (M-F. Fortin 1996 : 93). Nous inscrire dans cette logique argumentative implique que la toile de fond théorique de la présente étude doit être clairement déterminée. A ce sujet, nous convoquons deux théories linguistiques : il s'agit, notamment, de la théorie structurale et de la grammaire du sens selon la conception de P. Charaudeau (1992). Nous en faisons une présentation succincte, dans l'optique de montrer la pertinence de leur choix.

\subsubsection{La grammaire structurale}

La grammaire structurale décrit la combinatoire des unités syntagmatiques pour apprécier la compatibilité des mots ou groupe de mots et le degré de cohésion de ceux-ci dans la linéarité phrastique. Le volet syntaxique coïncide avec le volet sémantique pour faire ressortir le sens de phrase. Le choix de ladite théorie servira à analyser les relations syntaxiques et sémantiques des 
différentes parties du discours utilisées dans la construction de la phrase Vieux père, voilà ton petit, ce qui permettra d'en dégager le sens dénoté, lequel sera implicitement comparé au sens contextuel dans les actes locutoires des Ivoiriens.

\subsubsection{L'apport de la grammaire du sens}

Concernant la grammaire du sens, mentionnons qu'elle a été mieux élaborée par sous Patrick Charaudeau (1992). Pour cet auteur, l'étude de la langue ne saurait se limiter aux dimensions morphologique, syntaxique et aux diverses valeurs qui en découlent. Il affirme que "le langage est ce matériau qui permet à l'homme de construire du sens dans le monde tout en entrant en communication avec les autres. Le langage est donc à la fois sens, expression et communication. Il n'est pas successivement l'un et l'autre, mais les trois à la fois » (1992: 4). Partant de cette observation, le linguiste détermine l'objet de la grammaire de sens :

« La grammaire de sens doit donc s'intéresser à décrire les faits de langue en fonction :

-des intentions du sujet parlant qu'ils sont susceptible d'exprimer, ce qui exige que les catégories de la langue soient regroupées autour de ces intentions,

- des enjeux communicatifs qu'ils révèlent, ce qui exige que les différents systèmes de la langue soient traités du point de vue du sens,

- des effets de discours qu'ils peuvent produire » (Idem, 04)

L'expression «vieux père, voilà ton petit» nous place dans un contexte interactionnel mettant en scène le locuteur «ton petit» et l'interlocuteur « vieux père ». Or, conformément à la théorie sémantique d'A. H. Gardiner (1989), dans toute interaction, le locuteur manifeste une intention qui consiste non seulement à attirer l'attention de l'interactant sur un certain état de chose, mais aussi à le faire réagir à cet état de chose. Ainsi, pour lui, toute production discursive est un ensemble de mots révélant une intention (1989 : 91). Par la pratique de la grammaire du sens, il s'agira de déterminer l'intention de «l'agent volontaire» Charaudeau (Op. cit., p742), les enjeux communicationnels et les effets de sens de cette allocution. Suite aux précisions relatives au cadre théorique, nous présentons la partie méthodologique du présent travail.

\subsection{Aperçu méthodologique}

Dans le cadre de la présente étude, nous avons fait une immersion dans le milieu des locuteurs du français basilectal, c'est-à-dire ceux qui emploient 
couramment l'expression, objet de notre réflexion. L'enquête a été réalisée à l'aide d'une enregistreuse à clé USB. Il s'agissait de réaliser des enregistrements dans des espaces régulièrement fréquentés par cette catégorie de locuteurs composée de petits mécaniciens, de ferrailleurs, de "'gnabros", de "blakis", , de "balanceurs" ${ }^{4}$ " et de chauffeurs des "gbakas" en Côte d'Ivoire. Dans le cadre de l'enquête, notre immersion dans ces sphères, où règne une véritable course aux gains, s'est présentée sans grande difficulté dans certains lieux comme ceux des ferrailleurs, des blakis, etc ; par contre, dans celui des gnabros, nous avons déboursé un peu d'argent pour motiver nos interlocuteurs avant le déroulement de l'enquête.

Les questions s'articulaient toujours autour des points suivants :

- D'où vient l'expression Vieux père voilà ton petit / ton fils?

- A qui renvoient Vieux pères et petits?

- Combien de sorte de Vieux père existe-t-il ?

- Où peut-on localiser les Vieux pères?

- Que signifie Vieux père voilà ton petit / ton fils?

- Les Vieux pères comprennent-ils le sens du message?

- Quelle est l'attitude des Vieux pères face au message?

Par souci d'efficacité, nous avons étendu l'enquête sur deux (2) mois (novembre et décembre 2017) et limité à trois (3), le nombre de personnes à interroger, vu la diversité des espaces d'enquête. Ainsi, pour recueillir le point de vue des petits mécaniciens, nous avons ciblé Yopougon-kouté, rue des garages, face à l'église Catholique saint Mathieu, pour la simple raison qu'il s'agit pour nous d'un lieu non étranger parce que nous avions l'habitude d'y faire réparer notre véhicule.

Concernant les ferrailleurs, nous nous sommes rendu sur l'espace occupé par la ferraille d'Abobo, un quartier périphérique de la commune d'Abidjan. La motivation qui sous-tend ce choix s'explique par le fait qu'il constitue un lieu grouillant de locuteurs du français basilectal et du nouchi.

Quant à l'entretien avec les gnabros et les balanceurs, il a eu lieu dans leur «fief», précisément à Adjamé 220 logements, pendant leurs heures creuses (12 h-13h).

\footnotetext{
2 - Le terme "'gnabro" est provient de la langue malinké et se décompose de la sorte désigne : "'gna" qui veut dire "mes" et "bro"' qui désigne 'bras. Dans ce contexte, il signifie le gain obtenu à l'aide de mes bras, par force physique.

3 - Le mot "blakis" est une appellation désignant les commerçants du marché noir de la commune d'Adjamé, un lieu de tous les trafics.

4 _ 'Balanceur'” est la dénomination qu'on attribue aux apprentis des ''gbakas'. Il s'agit des mini-cars de transport en commun sur les lignes Yopougnon / Ajamé et Abobo /Adjamé, noms des communes d'Abidjan
} 
L'espace blakis a été le lieu de clôture de notre enquête. Il est situé à Adjamé-mosqué. C'est un lieu où règne une grande agitation de petits commerçants et d'autres locuteurs à la recherche de gains faciles.

Avant de présenter le résultat de l'étude qui se fonde sur la synthèse des données recueillies sur le terrain, il convient de procéder à l'analyse de l'énoncé, afin de ressortir sa signification dans le français standard.

\section{Analyse de Vieux père, voilà ton petit dans le français standard}

Sur le plan structural, la phrase est constituée de deux blocs syntagmatiques juxtaposés qui présentent une double partition du point de vue informationnel : une portion thématique Vieux père et une autre prédicative voilà ton petit, la notion de prédication devant être appréhendée comme le pôle pivot de la détermination du sens de la structure de la phrase. La portion thématique est composée de l'adjectif qualificatif vieux antéposé au substantif père. En effet, comme l'ont déjà remarqué Baylon et Fabre, « du point de vue sémantique, l'adjectif exprime une qualité ou une relation alors que le substantif désigne, comme son nom l'indique, une substance » (1989:44). Contrairement au déterminant qui observe une position syntaxique figée dans son rôle d'actualisation du substantif (le monde / ${ }^{*}$ monde le), l'adjectif épithète jouit, au contraire, d'une mobilité syntaxique. Dans sa fonction d'épithète, postposé ou antéposé directement au substantif accompagné, il n'influence généralement pas le sens du lexème auquel il est rattaché, sauf dans certains contextes stylistiques où la permutation positionnelle produit une variance sémantique. Selon C. Baylon et P. Fabre (1989: 47), « un certain nombre d'adjectifs connaissent une opposition de place pertinente du point de vue sémantique, l'antéposition marquant une tendance à l'affectivité ». En guise d'illustration, les auteurs de la grammaire systématique de la langue française donnent quelques exemples :

- Un grand homme / un homme grand

- Un simple soldat / un soldat simple

- Un vieil ami / un ami vieux (un homme talentueux, de grande valeur,

Dans le premier énoncé, l'antéposition de l'adjectif grand fait allusion à la qualité intrinsèque de l'individu ; il s'agit d'une personne talentueuse, possédant de grandes valeurs. Or quand l'adjectif grand est postposé, il se rapporte à la stature ou la taille de l'individu.

Antéposé dans le second énoncé, l'adjectif simple décrit la situation d'un soldat non gradé. En position finale, il s'agit d'un soldat qui ne jouit pas de tous ses sens.

Dans le dernier énoncé, un vieil ami renvoie à un ami de longue date alors qu'un ami vieux évoque l'âge de la personne.

Nous inspirant des variances sémantiques résultant de l'opposition pertinente de place des adjectifs décrits ci-dessus, nous procédons à analyse 
de la posture syntaxique de vieux dans l'expression vieux père. Comparativement au sens de «vieil ami / ami vieux», nous dirons que le qualificatif vieux dans vieux père fait allusion à une personne qui a été père, dans le sens de géniteur, il y a bien longtemps, à telle enseigne qu'il possède beaucoup d'expériences en tant que père. Dans le domaine religieux, il renvoie au statut de Doyen d'un membre supérieur d'Ordre religieux, remarquable par la sainteté de sa vie et reconnu par l'Eglise comme témoin d'une autorité exceptionnelle. Dans ce contexte, l'antéposition de l'adjectif vieux permet de mettre l'accent sur l'expérience accumulée dans sa fonction d'Abbé. Par contre, un «père vieux» renvoie à un géniteur ou à un ecclésiastique appartenant à une frange d'âge avancé. Ainsi, dans le français standard, vieux père renvoie soit à un individu riche d'expériences dans son rôle de chef de famille du fait qu'il est père de longue date, soit au statut de Doyen d'un religieux.

$\mathrm{Au}$ terme de l'analyse du premier segment énonciatif, nous nous focalisons maintenant sur le second, c'est-dire la partie prédicative de la phrase, celle qui représente ici le pôle important dans la détermination sémantique de la concaténation syntagmatique. Pour ce faire, nous nous centrons sur les propriétés sémantiques de voilà (constituant noyau dudit segment) telles que décrites dans la littérature grammaticale classique.

Dans les grammaires traditionnelles, les analyses consacrées au morphème voilà se limitent à quelques paragraphes. Le bon usage de Maurice Grevisse (1992) décrit l'usage de voilà en ces lignes : «Voici, voilà sont traditionnellement rangés au nombre des prépositions. Mais on pourrait avec raison les considérer comme des adverbes de lieu servant à présenter, comme des présentatifs » (1992: 956). L’emploi du lexème présentatif est aussi observable dans la Grammaire du français publiée chez Larousse par J-C. Chevalier et $a l$., (1964). En effet, ces auteurs soulignent que voilà a pour «seul rôle de présenter des noms, ou leurs équivalents » (1964 :84). Quant à M. Arrivé et al., ils n'emploient pas le terme présentatif mais affirment que voilà et voici appartiennent à la catégorie «des mots ou expressions qui permettent de désigner quelqu'un ou quelque chose en rapport avec une situation » (1986 : 565). Considéré sous cet angle, l'usage de voilà permet d'indiquer ici la présence de ton petit, groupe syntagmatique pouvant avoir pour substitut ton fils dans la situation communicationnelle examinée. Ainsi, sur le plan syntaxique et sémantique, l'on pourrait établir l'équivalence suivante : «Vieux père, voilà ton petit $=$ vieux père, voilà ton fils = vieux père, ce petit est ton fils = vieux père, c'est ton petit ou vieux père, ton petit est arrivé / venu ». Compte tenu des descriptions et des explications qui précèdent, l'on peut déduire que le sens général de l'énoncé consiste à présenter deux locuteurs observables dans le même espace de communication. Vieux père, voilà ton petit signifie alors dans le français courant que "le fils / le petit du père est 
arrivé ou ce petit est ton fils"; la distribution du présentatif voilà met alors en évidence la position d'éloignement du petit par rapport à celle du vieux père. Si le sens de l'expression peut s'appréhender ainsi dans le français standard, quelle acceptation observe-t-on dans le paysage conversationnel des Ivoiriens?

\section{Description sémantique de Vieux père, voilà ton petit dans le contexte ivoirien}

La description sémantique de l'énoncé dans le contexte ivoirien se déclinera en deux parties : d'une part, ayant remarqué que l'énoncé renvoie à une situation qui implique au moins deux interlocuteurs (vieux père et ton petit, le second étant l'énonciateur), le premier point de cette section servira à examiner les référents réels impliqués dans ces fragments interlocutifs. Pour ce faire, l'analyse s'appuiera sur la valeur contextuelle des syntagmes utilisés dans la constitution de l'énoncé tout en tenant compte des données recueillies lors de l'enquête. De l'autre, l'étude s'évertuera à décrire et à expliquer les différents sens et effets de sens qui en résultent.

\subsection{Etude référentielle de Vieux père » et de ton petit / tes petits}

Dans cette section, la notion de référents renvoie à un [- animé] ou un [+ animé] du monde qui fonde le sens de l'expression. Le sens d'une expression, pour reprendre J. C. Anscombre (2001 : 4), est ce qui permet d'identifier son référent car " tout signe linguistique n'existe que dans son fonctionnement social» (M.-A. Paveau et G-E. Sarfati, 2003 : 168). Ainsi, analyser les référents de l'expression vieux père / vieille mère dans le contexte ivoirien, revient à «donner des directives générales sur son utilisation pour référer à ou mentionner des individus ou des objets particuliers » (P. F. Strawson, 1971 : 18).

Analysés précédemment comme deux syntagmes indépendants, chacun étant doté d'une autonomie syntaxique et sémantique, les deux termes vieux et père observent une mouvance catégorielle dans le parler des Ivoiriens. Dans le français de Côte d'Ivoire, le lexème vieux n'est plus utilisé en tant qu'épithète décrivant la qualification du substantif père. Dans le contexte ivoirien, la combinaison de vieux et de père aboutit plutôt à la formation d'un nom composé. Dans le français standard, le groupe lexématique vieux père et l'unité père possèdent le même trait sémantique dans la mesure où sur le plan sémique, on a toujours la présence d'un géniteur ou d'une personne investie d'une mission ecclésiastique. Or, dans le contexte ivoirien, vieux père et père n'ont pas le même trait sémantique parce que, du point de vue référentiel, le syntagme vieux père renvoie maintenant à une nouvelle image, acquiert un autre sens, les deux paradigmes ayant subi le phénomène de désémantisation et de recatégorisation. Ce groupe 
syntagmatique ne fait plus allusion ni au statut de Doyen d'un Abbé, ni à un père de longue date dans le sens de géniteur comme précédemment décrit ; mais dans le contexte ivoirien, il renvoie à une catégorie d'individus bien déterminés. L'opposition observée dans le couple vieux père / petit permet ainsi d'opérer une comparaison sous-jacente dans laquelle est mise en saillance l'identité du locuteur qui appartient à une catégorie de personnes appelée petits, une catégorie infériorisée et positionnée au bas de l'échelle sociale par rapport à celle de vieux père. En d'autres termes, selon les personnes interrogées lors de l'enquête, les dénominations vieux père et ton petit /tes petits implicitent un niveau de clivage bicéphale. D'un côté, la catégorie des vieux pères / vieilles mères et de l'autre, celle des petits / enfants / fils. Le sens de toute réalisation énonciative étant consubstantiel à son contexte de production, nous allons analyser la structure hiérarchiquefonctionnelle des référents auxquels renvoient les groupes morphématiques vieux pères et tes petits en tenant compte des facteurs extralinguistiques qui déterminent leur actualisation. Pour la clarté de l'analyse, nous procédons à une description séparée de chaque bloc énonciatif. Ainsi, nous étudierons la composition catégorielle de vieux père / vieille mère en premier lieu et celle de petit $(s) /$ fil $(s)$ en second lieu.

\subsubsection{Les référents dans l'énoncé vieux pères / vieilles mères}

Nous précisons que la description de référents des unités vieux pères / vieilles mères qui va suivre ressort du résultat de l'enquête. Il faut dire que ces suites syntagmatiques présentent une stratification observable en trois sous-catégories : Les vieux pères de la catégorie (1) ; Les vieux pères de la catégorie (2) et Les vieux pères de la catégorie (3). L'explication des trois catégories spécifiant la structure hiérarchique de la catégorisation des vieux pères s'appréhende de la façon suivante :

- Les vieux pères de la portion (1) représentent les catégories des Ivoiriens les plus riches. Ceux-ci sont en nombre restreint. Les Ivoiriens de cette catégorie sont économiquement très forts à telle enseigne qu'ils peuvent s'offrir tous les voyages internationaux et toutes les catégories de matériels de confort. Ils habitent les quartiers huppés (Deux Plateaux, Riviera, CocodyAngré, Cocody-Vallon, etc.).

- Les vieux pères de la portion (2) regroupent la catégorie des Ivoiriens d'aisance moyenne. Ceux-ci ont une bourse moins consistante que celle des personnes de la classe (1). Ils sont logés dans les mêmes quartiers que les précédents mais occupent des maisons à loyer moins élevé ; on peut aussi les localiser dans les quartiers résidentiels de ceux des portions (3).

- Les vieux pères de la portion (3) représentent la frange d'Ivoiriens économiquement faibles. Concernant la satisfaction de leurs besoins, ceux-ci accordent une priorité aux dépenses incompressibles. Ignorant l'existence du 
superflu financier, ils résident dans certains quartiers comme Yopougon, Koumassi, Port- Bouët, etc.) dans des maisons à loyer modéré.

De cette hiérarchisation, nous retenons que contrairement à la conception classique où les unités « vieux pères / vieilles mères » désignent un père ou une mère de longue date, ces suites sont plutôt utilisées pour catégoriser socialement les Ivoiriens qui vivent dans l'opulence. Sur ce, examinons le cas de « mon petit / mes petits ».

\subsubsection{Les référents de l'entité des « petits / fils »}

Les deux unités petits et fils renvoient chacune à une catégorie d'Ivoiriens. Il s'agit, notamment, de ceux identifiables dans la frange des pauvres. A cet égard, notons qu'on peut aussi distinguer un clivage à l'intérieur de ce groupe : les moins pauvres et les indigents.

- Les pauvres représentent la couche des locuteurs les plus nombreux. Certains, pour la plupart, sont en quête d'emploi. D'autres sont des débrouillards du secteur informel. Le manque criard de moyen financier les amène à satisfaire à peine leurs besoins.

- Cette catégorie est composée d'une autre sous-catégorie : les indigents. Ceux-ci sont dans l'incapacité de satisfaire à leur nécessité vitale. Certains vivent dans les quartiers dont la dénomination connote la misère, où les constructions jouxtent les dépôts d'ordures. Nous faisons allusion, notamment, aux appellations comme «mon mari m'a laissée», « tomber mourir » (quand on tombe, on meurt: quartier où les habitations sont construites sur des pentes très dangereuses à telle enseigne que la grande saison de pluie cause toujours de nombreux morts du fait d'éboulements), « sicobois » (presque toutes les habitations, de véritables bric-à-brac de fortunes sont en bois), etc. D'autres vivent dans des ghettos à la périphérie des quartiers précédemment cités. Résignés à leur sort, plusieurs d'entre eux s'adonnent à la drogue, à l'alcool, comme s'ils voulaient précipiter leur mort.

Dans le quotidien, ce monde de démunis côtoie régulièrement ceux de la catégorie des économiquement forts. Du 'frottement', des deux catégories sociales est née l'expression 'vieux père / vieille mère, voilà ton petit / ton fils ". Etant donné que "toute énonciation, même sous sa forme écrite figée est une réponse à quelque chose » (M. Bakhtine, 1977 : 106), et que chaque forme, selon Paveau et Sarfati (2003:168), est porteuse de sens et que ce sens est issu d'une production sociale, nous nous posons la question suivante : quelles sont les charges sémantiques et les effets de sens "portés" par l'énoncé ?

\subsection{Effets de sens de vieux père, voilà ton / tes petit (s)}

Parlant de la notion d'effets de sens, P. Charaudeau et D. Maingueneau affirment qu'elle « est liée dès son origine à la notion de discours, bien qu'elle 
fasse l'objet de différentes définitions selon la théorie dans laquelle elle s'inscrit » (2002 : 207). Dans la conception de G. Guillaume (1964), cité par ces auteurs, les effets de sens correspondent à l'infinie variété des valeurs que les unités linguistiques peuvent acquérir dans le discours, selon le contexte dans lequel elles s'inscrivent. En d'autres termes, les effets de sens ne sont que le résultat des valeurs que le discours attribue au signifiant en langue (Ibid.). En évoquant la notion d'effets de sens, nous entendons faire ressortir la valeur discursive de l'énoncé, laquelle est en corrélation avec «la visée d'effet particulière » (Ibid.) animant le locuteur.

Après la spécification référentielle des syntagmes vieux pères et tes petits plus haut, l'analyse des effets de sens se focalisera sur la description de la valeur discursive du présentatif canonique voilà dans son rapport syntaxique avec l'adjectif possessif ton, ces deux constituants jouant un rôle capital dans la détermination sémantique de la séquence énonciative. En effet, dans l'acception traditionnelle, la propriété sémantique de voilà se résume à une simple fonction de présentation, de monstration. Il s'agit là de conceptions incomplètes parce qu'elles occultent les caractéristiques ou le mode de présentation de l'élément dans le rapport de présentant / présenté. En d'autres termes, elles n'apportent pas de précision sur l'identité ou sur les qualités de l'élément présenté. La non-prise en compte de ce paramètre explique la position de G. Moignet à préférer la dénomination de « verbes d'existence /... / accompagnés d'un élément de référence situationnelle » (1981: 279) plutôt que le terme présentatif. Or, la perspective analytique qui cadre avec la présente section transcende la fonction de simple présentation véhiculée par voilà. Certes, voilà est une entité linguistique dont le rôle consiste à présenter ; mais à présenter qui et sous quelle identité ? Dans quelles intentions ? Sur ces aspects, la fonction de monstration inhérente à ce syntagme "reste muette ». C'est sur ces différentes préoccupations l'analyse de P. Charaudeau (1992) apporte des éclairages. En effet, dans la Grammaire du sens et de l'expression, cet auteur indique que "la présentation est l'opération linguistique qui correspond à l'intention de déterminer le mode d'existence d'un être (ou d'un processus) /.../» (1992 : 302). Pour notre étude, l'aspect digne d'intérêt dans cette citation concerne le volet évoquant l'intention comme le fondement de la présentation. Charaudeau le corrobore clairement en indiquant que ce qui importe, c'est moins l'acte de présentation que le mode de présentation. Dans sa perception, «il faut dire à quelle intention d'expression correspond la présentation, puis quelles sont, en relation avec les marques qui permettent d'exprimer cette intention, les spécifications de cette intention » (Ibidem, p. 302). Sachant que le ressort de l'action est l'intention, et que c'est elle qui impulse et qui donne sens ou valeur à l'action, l'angle analytique de l'auteur nous conduit à l'interrogation suivante : dans 
l'acte locutoire vieux père, voilà ton petit, quelles sont les intentions réelles du locuteur? Quels effets de sens recouvre l'énoncé dans le contexte ivoirien?

\subsubsection{L'effet de rapprochement dans l'énoncé vieux père, voilà ton petit}

Pour donner du sens à un acte locutoire, il convient d'appréhender les intentions, les visées et les buts assignés à l'action. Dans cette logique argumentative, le sens d'un énoncé est l'idée qui lui correspond dans l'esprit de l'énonciateur. Le sens d'un acte locutoire correspond donc à la visée subjective ou à l'intention de celui-ci ; c'est pour cela dans certains contextes langagiers, l'usage de la locution adverbiale dans l'intention de est synonyme de dans le sens de. Le balisage explicatif de la convergence sémantique des deux unités est nécessaire du fait qu'il permet de mieux éclairer la suite de l'étude. Sur ce point, une question se pose : en quoi l'énoncé recouvre-t-il un sens de rapprochement?

Le sens de rapprochement véhiculé par l'expression est-il en lien avec l'intention du locuteur à présenter son identité, celle qui porte les stigmates de la condition d'existence difficile de la catégorie sociale des petits comme décrite supra? L'explication pour ce volet sémantique nécessite que nous nous focalisons sur l'analyse de l'identité du présenté, une analyse qui se fera en s'appuyant sur la distribution de l'adjectif possessif ton. Dans la grammaire méthodique $d u$ français, M. Riegel et al. (2004 : 204-205) affirment que l'adjectif possessif est une sous-classe de déterminant défini dont la propriété consiste à actualiser le substantif en lui donnant un contenu de possession et d'appartenance. Dans le contexte-ci, l'adjectif possessif ton crée donc une relation d'appartenance entre petit et vieux père $($ ton petit $=$ petit de toi $=$ petit de vieux père). A cette propriété s'ajoute celle résultant de l'étude des possessifs menée par P. Garde (1998). En effet, dans La grammaire russe, cet auteur fait savoir qu'à la relation de possession et d'appartenance véhiculée par les déterminants possessifs, s'adjoint une autre propriété sémantique : celle de la « dépendance » (P. Garde, 1998 : 263). Ainsi, à travers la suite syntagmatique ton petit $=$ petit de toi $=$ petit de vieux père, il se greffe une idée de dépendance sur cette relation de possession-appartenance.

Souvent aussi, dans certaines séquences locutoires, le syntagme ton petit est renforcé par ton bon petit ou simplement remplacé par ton fils / ton enfant si bien qu'on peut établir une équivalence sémantique entre ton petit et ton fils. Une telle substitution permet de positionner clairement le volet sémantique de l'énoncé sur le plan de la filiation artificielle. Dans cet ordre d'idées, la valeur de rapprochement est combinée avec l'idée de dépendance qui conditionne le rapport entre le fils et le père. A partir de l'instant où la juxtaposition des deux blocs énonciatifs donne l'existence à un contexte de filiation factice, le locuteur (mon petit) et l'interlocuteur (vieux père) sont 
présentés comme deux personnes proches appartenant à une même famille. La création d'un contexte de lien de parenté entre deux interactants, très éloignés, en général l'un de l'autre, s'interprète ici comme une stratégie d'approche utilisée par le locuteur pour atteindre un objectif. Lequel ? Le point suivant clarifie cet implicite.

\subsubsection{De l'effet de rapprochement à celui de requête}

Dans un acte locutoire, selon P. Charaudeau (1992), alors que l'usage de l'adjectif possessif mon signale, de façon générale, que le locuteur endosse la responsabilité de la dépendance, ou que le destin de celui-ci est lié à celui de l'interlocuteur, l'emploi des possessifs ton et son permettent au locuteur de se dégager de la responsabilité d'une appartenance quelconque et de souligner que c'est l'interlocuteur ou le tiers qui doit endosser la responsabilité (1992 : 207). Ainsi, dans vieux père, voilà ton petit, l'usage de ton montre que l'agent volontaire ton petit se décharge de toute responsabilité qu'il veut faire endosser au vieux père. L'évocation d'un contexte de lien de familiarité hypocristique constitue donc un stratagème dont le but est de manipuler l'esprit de l'interlocuteur pour rendre celui-ci réceptif à la charge que le premier lui demande subtilement de porter. Dans la section antérieure relative à l'analyse référentielle de la catégorie sociale des petits, nous avons fait mention de la diversité de difficultés qui entravent l'épanouissement des personnes de cette tranche sociale. Il s'agit, entre autres, de l'impossibilité d'assurer le minimum vital comme celui de se nourrir convenablement faute de moyen. Face à cette situation, la rue devient un espace idéal pour obtenir les faveurs ou le soutien des vieux pères et des vieilles mères. L'expression Vieux père, voilà ton petit, créée dans cette intention, sert donc non seulement à présenter la situation d'existence difficile du locuteur mais elle constitue aussi une stratégie utilisée pour chercher à "déstabiliser", émotionnellement les vieux pères au point de les amener à réagir positivement. Dès lors, l'énoncé véhicule un sens de requête, de demande. En se présentant sous l'étiquette de petit, le locuteur catégorise ce syntagme comme un marqueur diminutif à valeur péjorative dont l'usage lui permet d'exposer sa condition sociale difficile. En fait, la situation est celle d'une demande d'aumône que l'on rencontre aussi ailleurs sous d'autres formes. Dans ce contexte, la distribution du présentatif voilà et de ton petit permet de conférer la valeur axiologique de l'auto-apitoiement à l'énoncé. Appréhendé sous cet angle, l'énoncé pourrait se transformer ainsi : Vieux père, voilà ton petit malheureux ou Vieux père aidez-moi, je suis malheureux. L'expression épouse par ce fait « un effet de dramatisation » (P. Charaudeau, 1992 : 318), lequel consiste à susciter de la compassion chez l'interlocuteur (vieux père) tout en espérant que celui-ci fasse preuve d'une marque de solidarité. Dans cette optique analytique, petit est en usage bi-fonctionnel dans le sens où il peut être 
décrit selon la conception de C. Kerbrat-Orrechioni comme un «adjectif subjectif » et « affectif » (1980: 84). L'évocation de la notion de subjectivité s'explique par le fait que, se présentant sous le vocable petit, le locuteur s'inscrit dans son énoncé en établissant une comparaison implicite entre la classe des vieux pères et celle des petits. Cette "coloration" subjective est accompagnée d'une dose d'affectivité puisque l'expression recouvre une valeur " vocative », laquelle permet à l'énonciateur de se présenter comme un être à plaindre, quelqu'un qui pleure sur son sort pour attirer la compassion du vieux père.

\section{Conclusion}

En somme, il convient de retenir qu'en Côte d'Ivoire, la langue française continue de "faire sa mue" sur les plans morphologique, syntaxique et sémantique. Dans le français standard, l'énoncé vieux père, voilà ton petit décrit une situation de communication où sont présentés deux interlocuteurs. Le premier, vieux père, fait allusion au statut de père de longue date par rapport au second. Entre les deux actants, il existe une relation de familiarité, le petit dépendant du vieux père. Dans le parler des Ivoiriens, le syntagme vieux père se distribue en tant qu'un substantif composé, renvoyant à une nouvelle réalité. L'énoncé est employé pour décrire ou pour suggérer une comparaison implicite entre deux classes sociales. L'une (celle des vieux pères) est composée de personnes qui vivent dans l'opulence alors que l'autre (ton / tes petits) représente celle des individus dont l'existence connote la pauvreté. L'emploi récurrent de l'expression en question devient un moyen utilisé par les personnes de la deuxième catégorie pour s'auto-apitoyer, pour se présenter comme des malheureux ou des personnes à plaindre dans le but de déclencher le sentiment de compassion des vieux pères afin d'obtenir de l'aide de leur part. Ainsi, à la valeur de rapprochement émanant de l'énoncé s'adjoint celle d'une demande d'aide.

\section{References:}

1. ARRIVE M.; GADET F.; GALMICHE M. (1986). La grammaire d'aujourd'hui - guide alphabétique de linguistique française, Paris, Flammarion.

2. BAKHTINE M. (1977). Le marxisme et la philosophie du langage, Paris, Minuit.

3. BAYLON C. ; FABRE P. (1989). Grammaire systématique de la langue française, Paris, Nathan.

4. BOHUI H.D. (2015). Petit recueil d'ivoirismes, Abidjan, Le GRAAL.

5. CHARAUDEAU P. (1992). Grammaire du sens et de l'expression, Paris, Hachette Education. 
6. CHARAUDEAU P.; MAIGUENEAU D. (2002). Dictionnaire de l'analyse du discours, Paris, Editions du Seuil.

7. CHEVALIER J.-C. ; ARRIVE M. ; BLANCHE-BENVENISTE C. ; PEYTARD J. (1964). Grammaire du français contemporain, Paris, Larousse.

8. FORTIN M.-F. (1996). Le processus de la recherche: de la conceptualisation à la réalisation, Montréal, Décarie éditeur. M-F.

9. GARDE P. (1999). Grammaire russe, Paris, Institut d'Etudes slaves.

10. GARDINER A. H. (1889). Langage et acte de langage : aux sources de la pragmatique, Paris, P.U de Lille.

11. GOHIER C. (2000). Le cadre théorique. Dans T. Karsenti et L. SavoieZajc, Introduction à la recherche en éducation, pp. 99-125, Sherbrooke, Ed. du CRP.

12. GREVISSE M. (1992). Le bon usage : grammaire française, Paris, De Boeck Duculot

13. KERBRAT-ORECCHIONI, C. (1980). L'énonciation de la subjectivité dans le langage, Paris, Armand Colin.

14. KOUADIO N. J. (2008). "Le français en Côte d'Ivoire : de l'imposition à l'appropriation décomplexée d'une langue exogène ». Documents pour l'histoire du français langue étrangère ou seconde, 40-41: 179-197.

15. LAGERQVIST H. (2001). Introduction au français hors de France. Aalborg : Aalborg University Press.

16. MOIGNET G. (1981). Systématique de la langue française, Paris, Klincksieck.

17. PAVEAU, P. F. ; SARFATI, G.-E. (2003). Les grandes théories de la linguistique, Paris, Armand Colin.

18. RIEGEL M.; PELLAT J.-C.; RIOUL R. (2004). Grammaire méthodique du français, $3^{\mathrm{e}}$ Ed. Paris, P.U.F.

19. STRAWSON, P. F. (1971). Etudes de logique et de linguistique, Paris, Seuil. 